\title{
HIGH-SENSITIVITY SENSORS FOR MONITORING OF LEAD ATMOSPHERIC CORROSION
}

\author{
Kristýna Charlotte STRACHOTOVÁ, Milan KOUŘIL \\ ${ }^{1}$ University of Chemistry and Technology, Prague, Department of Metals and Corrosion Engineering, \\ Prague, Czech Republic, EU, strachok@vscht.cz, kourilm@vscht.cz
}

https://doi.org/10.37904/metal.2021.4237

\begin{abstract}
Thanks to the specific sensitivity of lead to volatile organic compounds (VOC), it is possible to use lead as an indicator of contamination of the indoor atmosphere of depositories of archives and museums where VOCs are typical pollutants. The classification of corrosion aggressiveness of indoor environments based on the determination of the corrosion rate of reference metals is given by the standard ISO 11844-1 which was recently supplemented with lead. Established techniques for determining the corrosion rate of lead are not sensitive enough to monitor the corrosive aggressiveness of the indoor environment. The aim of this project was to develop a promising, highly sensitive method for measuring the corrosion rate of lead in the indoor atmosphere. Innovative highly sensitive electrical resistance lead sensors have been developed for this purpose. At the same time, a low-cost alternative sensor working on the electrochemical principle was developed employingsingle frequency impedance measurement for indicating variations of surrounding air aggressivity. The sensitivity of such a sensor was observed to behigher than that of the traditionalelectrical resistance sensors.
\end{abstract}

Keywords: Lead, high-sensitivity sensor, atmospheric corrosion monitoring

\section{INTRODUCTION}

The specific case of lead corrosion, i.e. in the presence of organic pollutants (especially acetic acid vapours accelerate atmospheric corrosion of lead even at concentration $0.1-1 \mathrm{ppm}$ [1]), is typical for museum environments due to the presence of organic substances (with the concentration lower than $500 \mathrm{ppb}$ [2]) released from the enclosure materials [3]. Due to the high number of possible pollutants inside the building, the costs and technical problems associated with their monitoring, only relative humidity and temperature are monitored in most institutions. At the same time, real information about the aggressiveness of the given environment is necessary for effective protection of metal objects.

Atmospheric corrosion monitoring by means of electrochemical sensors is based on recording galvanic current flowing between to different metals exposed side-by-side in the air or on recording an impedance response of a pair of identical metal electrodes loaded with alternating electrical signal. The response of both the techniques depends on formation of an electrolyte layer, its thickness, conductivity and aggressiveness towards the metals. The evaluation of the aggressiveness of the environment is in real time and the measurement makes it possible to estimate the instantaneous corrosion rate [4-7]. Many types of electrochemical sensors are commercially available, but these sensors do not work with lead as the metal whose corrosion rate to be determined.

The rezistometric method according to ASTM G96 [8] and ISO 11844-2 [9] uses a sensor (consisting of a specific and a reference part) made of the monitored metal that is exposed to an aggressive atmosphere and the electrical resistance is measured, which increases with the loss of metal trace due to corrosion. Thanks to the integrated reference part in the sensor, changes of resistance due to changes in ambient temperature are compensated. The advantage of this technique is its applicability in a non-conductive environment, including 
atmosphere, and continuous monitoring of corrosion depth [4-6]. The sensitivity of electrical resistance sensors is limited by the thickness, width and length of the lead track. Thin lead foils are considerably expensive. At the same time, commercially available AirCorr electrical resistance sensors (with design A and E), used for laboratory tests in model conditions (accelerated corrosion tests), are not sensitive enough to measure the corrosion rate of lead in atmospheric conditions with real (very-low) pollutant contents and relative humidity values. Our goal was to develop sufficiently sensitive sensors for measuring the corrosion rate of lead in an atmosphere with a low concentration of pollutants and at low values of relative humidity, which will allow the classification of the aggressiveness of the atmosphere according to ISO 11844-1 [10]. Using a commercially available material (lead foil from Goodfellow with a thickness of $25 \mu \mathrm{m}$ ), which is an affordable variant, the sensitivity of electrical resistance sensors can be increased only by modifying the design of current sensors (the electrical resistance sensor with design $X$ developed in this investigation consisted of lead track twice as long aselectrical resistance sensor with design A). A low-cost alternative to the production of electrical resistance sensors is the production of an impedance sensor measuring the impedance between two identical electrodes formed by a system of parallel electrode plates, for the production of which expensive thin lead foils are not required.

\section{EXPERIMENTAL PART}

\subsection{Electrical resistance (ER) sensors}

The ER sensors employed in this investigation (Figure 1) were manufactured by means of the printed circuit technique using a photolithographic process. The pattern (design A (lead track length $107 \mathrm{~mm}$ ), E (lead track length $69 \mathrm{~mm}$ ) or $X$ (lead track length $192 \mathrm{~mm}$ )) is transferred to a glass-fibre board laminated with lead foil (97 wt \% Pb, 1.5 wt\% Sn, 1.5 wt\% Sb, Goodfellow, thickness of lead foil $25 \mu \mathrm{m}$ ) after selective removal (UV development) of a photosensitive layer applied to the lead foil. The transferred pattern then serves as a protective mask during the lead foil etching process. After the lead etching process is completed, the laminate board with a lead track (according to design $A, E$ or $X$ ) is thoroughly washed with distilled water and the protective mask is removed with ethanol. Subsequently, the reference part of the ER sensor is covered with a protective foil and the cables, which are then connected to the channels of the KEITHLEY control panel, are soldered with tin solder to the galvanically copper-plated contacts of the ER sensor. The contacts with the soldered cables and the foil protecting the reference part are finally coated with a two-component epoxy resin (UHU).

\subsection{Impedance sensor}

The impedance sensor developed and employed in this study (Figure 1) was prepared from 12 pieces of "L" shaped lead plates,thickness of lead sheet $1 \mathrm{~mm}$ ) parallel arranged, insulated on both sides with adhesive tape, connected with two copper wires and fixed with a two-component epoxy resin Epoxy 1000 (CT).

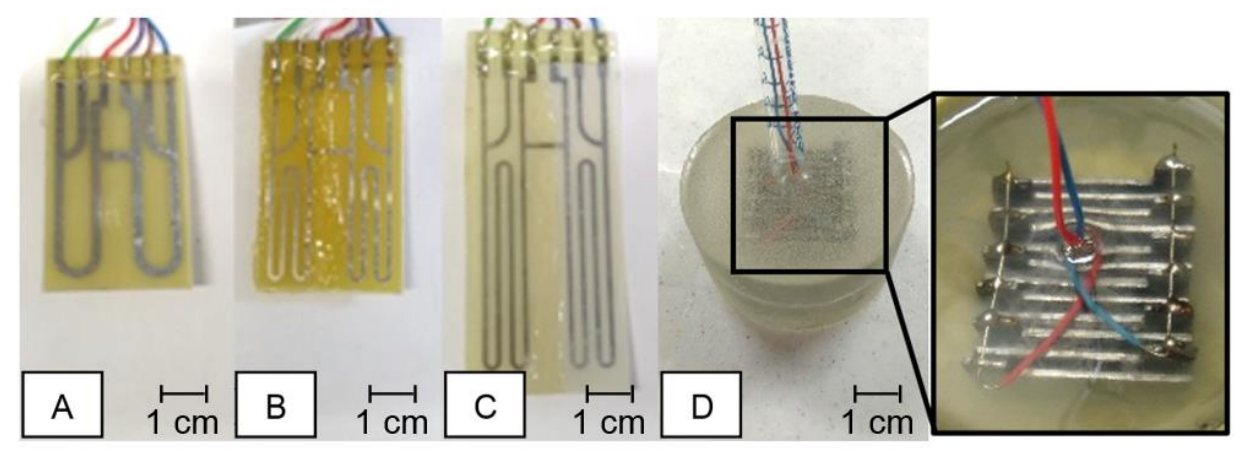

Figure 1 The ER sensors used in this investigation: ER probe with design $E(A)$, ER probe with design $A(B)$, newly developed ER probe with design $\mathrm{X}(\mathrm{C})$ and impedance sensor (D) 
Impedance measurement was performed manually, using the ELC-133A LCR meter connected with the sensor by means of attached copper wires.

\subsection{Laboratory exposures}

Sensors were exposed into the closed boxes with the volume of 10 litres with a ventilator and a beaker with $100 \mathrm{ml}$ of acetic acid solution.Corrosive atmosphere was provided by aqueous ( $100 \%$ relative humidity) or saturated $\mathrm{Na}_{2} \mathrm{Cr}_{2} \mathrm{O}_{7} \cdot 2 \mathrm{H}_{2} \mathrm{O}$ ( $\sim 52 \%$ relative humidity) solutions of acetic acid in various concentrations $(0.001$, 0.01 and $\left.0.1 \mathrm{~mol} \cdot \mathrm{I}^{-1}\right)$. Before exposure, the sensorssurfacewas dry grinded with abrasive wadding (3M ScotchBrite CF-MF).

\section{RESULTS}

\subsection{Response to changes in atmospheric conditions}

As shown in Figure 2 and Figure 3, all sensors reveal changes in exposure conditions. Readings from all sensors have shown humidity and concentration of acetic acid vapours dependency. For readings from the ER sensors, corrosion rate increased mostly when both factors (humidity and acetic acid vapours concentration) increased, while the impedance sensor responded also at lower humidity. According to Figure 2, the ER sensor with design A showed highest sensitivity to all exposure conditions. The ER sensor with design $E$ showed sensitivity to increase in acetic acid concentration even at low humidity, but its service life is shorter than that of the ER sensor with design $A$ and $X$. The presumption of an increase in the sensitivity of the ER sensor when extending the lead track (design X) has not been confirmed (Figure 2). For all the ER sensors, initial exposure conditions for starting detectable corrosion were identical $\left(0.001 \mathrm{~mol} / \mathrm{l}^{-1}\right.$ acetic acid aqueous solution).

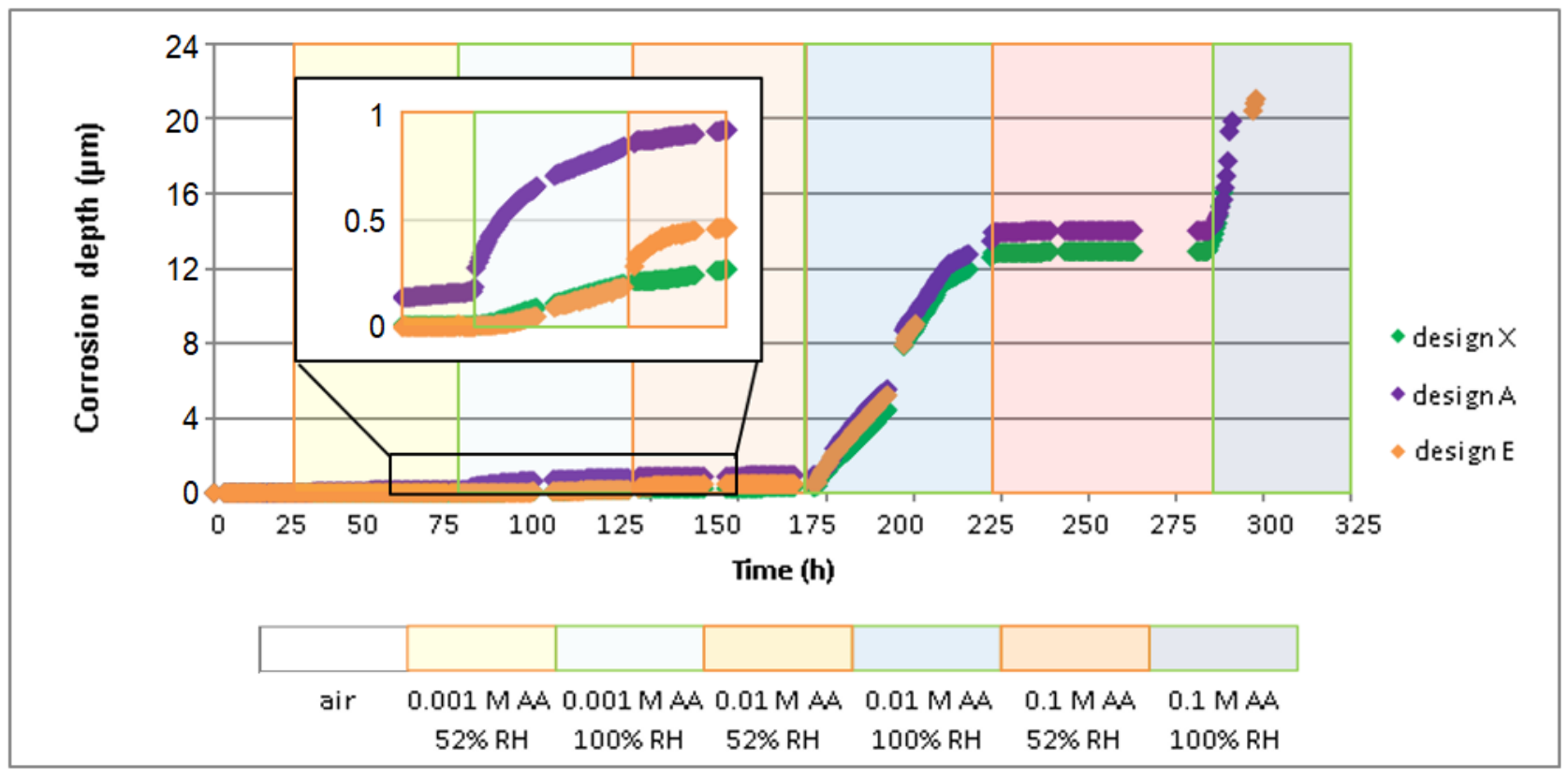

Figure 2 Exposure of ER sensors in corrosive atmosphere ( $\mathrm{AA}=$ acetic acid solution)

According to Figure 3, readings of capacitance measured by using the impedance sensor (notably at a monitored frequency of $100 \mathrm{~Hz}$ ) confirmed humidity changes during exposure.As can be seen from Figure 3 , 
during the first 72 hours of exposure of the impedance sensor in a corrosive environment, no impedance values were observed at the frequency of $100 \mathrm{~Hz}$ and $1 \mathrm{kHz}$ due to the overload of the LCR meter. Readings measured with an impedance sensor at a constant frequency of $10 \mathrm{kHz}$ showed a decrease in impedance values after adding acetic acid to the exposure (24 h). After the subsequent increase in humidity (while maintaining the same concentration of acetic acid) there was a significant decrease in impedance values while gradually increasing the values of capacitance for all monitored frequencies, a layer of corrosion products formed on the surface of the impedance sensor. With a further increase in the acetic acid concentration and a decrease in humidity, there was an expected decrease in capacitance values and an increase in impedance values; the corrosion rate is significantly higher than with a lower acetic acid concentration at the same humidity. The consequent increase in humidity increased the corrosion rate. The last increase in acetic acid concentration, while the humidity decreased, there was a significant increase in impedance values. Corrosion rate in $0.1 \mathrm{M} \mathrm{AA}, 52 \% \mathrm{RH}$ environment is higher than in $0.001 \mathrm{M} \mathrm{AA}, 52 \% \mathrm{RH}$ environment, but lower than in $0.01 \mathrm{M} \mathrm{AA}, 52 \% \mathrm{RH}$ environment.

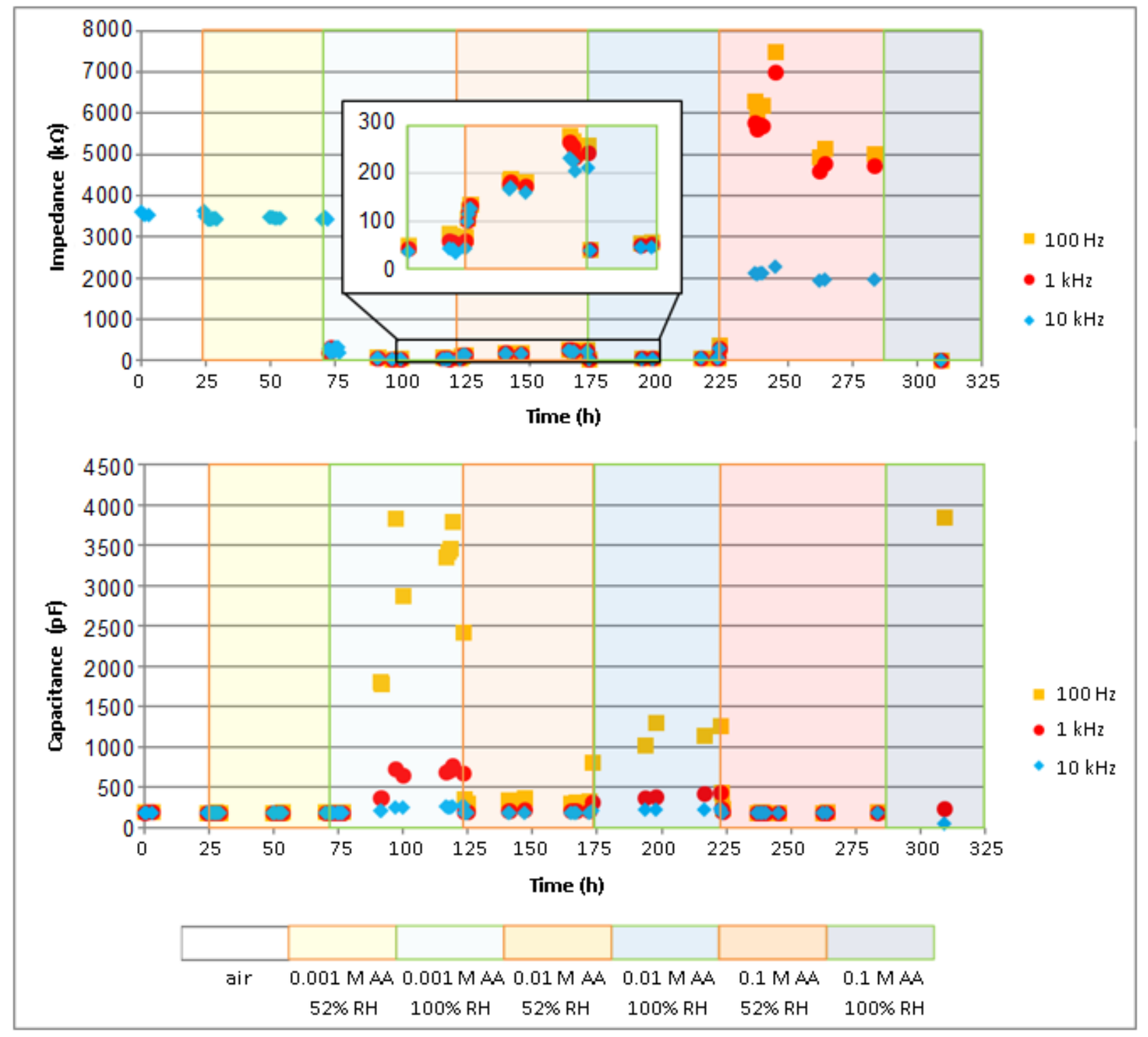

Figure 3 Exposure of impedance sensor in corrosive atmosphere ( $\mathrm{AA}=$ acetic acid solution) 


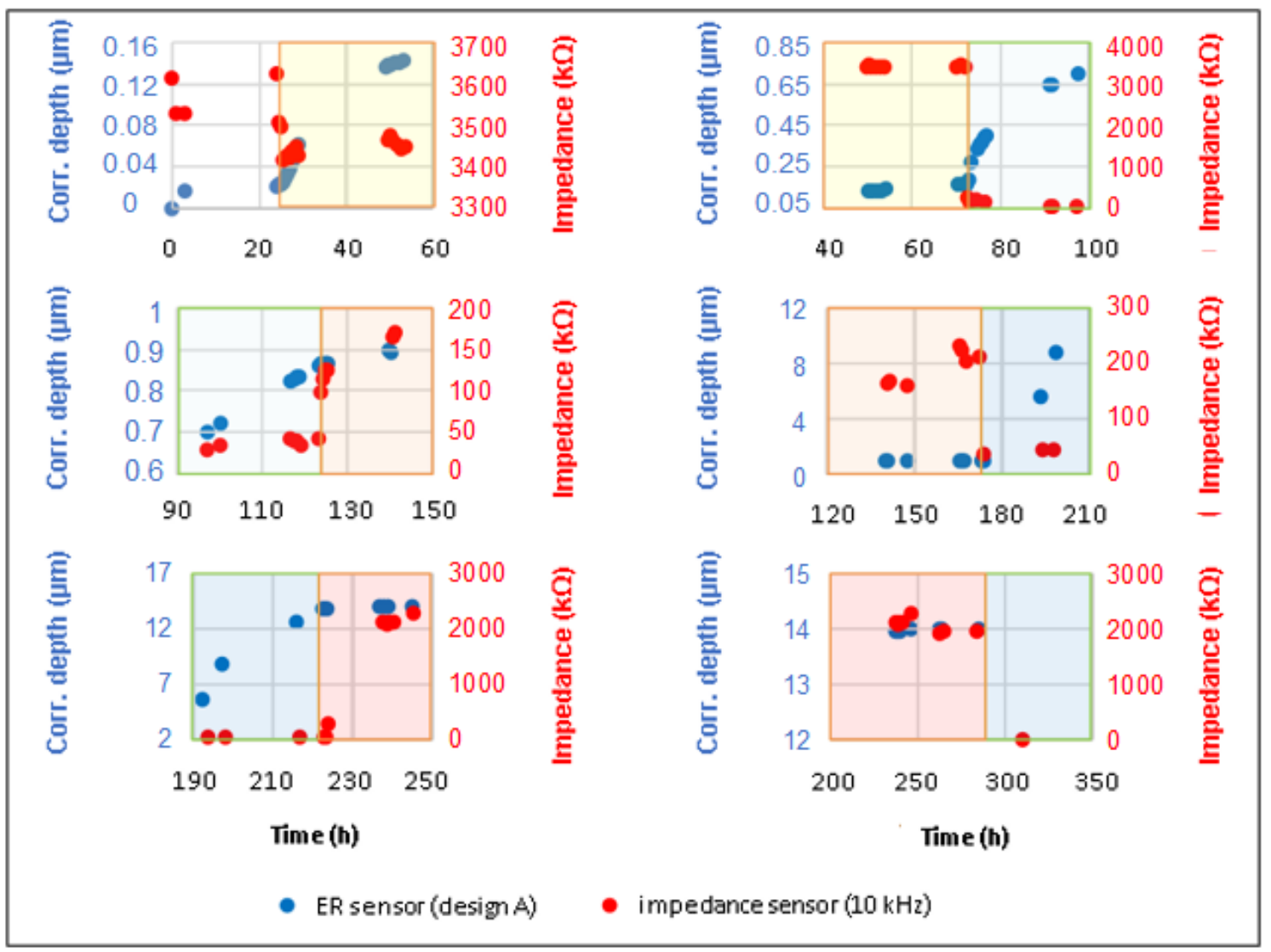

Figure 4 Time evolution of the resistance during the exposure of ER and impedance sensors in corrosive atmosphere

\subsection{Variability of results}

Table 1 and Table 2 show variability of measured values for ER and impedance sensors in different exposure conditions by means of average noise value of the measured data and the noise extreme values (maximum positive value of noise (MAX) and the lowest negative value of noise (MIN)). In very aggressive exposure conditions $(0.01 \mathrm{M} \mathrm{AA}, 100 \% \mathrm{RH})$ occurs in the ER sensor a significant increase the noise values of measured data (Table 1), on the contrary, the impedance sensor measures significantly more accurately at all monitored frequencies (Table 2).In all other exposure conditions, the data measured by the ER sensors are significantly less noisy than the data measured by the impedance sensor.According to Table 1, after the subsequent pollution of the atmosphere with acetic acid the measurement with the ER sensor with design $X$ is the most accurate. From the point of view of variability and reproducibility of measured data, ER sensors appear to be a more accurate method for corrosion monitoring of indoor atmospheres.

Table 1 Variability of measured values for ER sensors in corrosive atmosphere

\begin{tabular}{|c|c|c|c|c|c|c|c|c|c|}
\hline & \multicolumn{3}{|c|}{ design $E$} & \multicolumn{3}{|c|}{$\operatorname{design} \mathrm{A}$} & \multicolumn{3}{|c|}{$\operatorname{design} X$} \\
\hline & Onoise & MIN & MAX & Onoise & MIN & MAX & Onoise & MIN & MAX \\
\hline air & 0.000 & -2.9 & 5.3 & 0.000 & -2.0 & 2.2 & 0.000 & -2.9 & 5.3 \\
\hline $0.001 \mathrm{M}$ AA, $52 \% \mathrm{RH}$ & 0.000 & -11.1 & 6.8 & 0.149 & -1.5 & 2.4 & 0.000 & -1.9 & 0.5 \\
\hline $0.001 \mathrm{M} \mathrm{AA}, 100 \% \mathrm{RH}$ & -0.004 & -6.0 & 5.8 & 0.019 & -2.2 & 4.4 & -0.001 & -1.2 & 1.1 \\
\hline $0.01 \mathrm{M}$ AA, $52 \% \mathrm{RH}$ & -0.004 & -7.7 & 7.0 & -0.002 & -2.7 & 2.4 & 0.003 & -1.4 & 1.1 \\
\hline $0.01 \mathrm{M}$ AA, $100 \% \mathrm{RH}$ & -0.133 & -50.1 & 40.2 & 0.185 & -132.7 & 140.8 & -0.048 & -93.5 & 98.2 \\
\hline $0.1 \mathrm{M}$ AA, $52 \%$ RH & - & - & - & -0.121 & -0.7 & 0.6 & -0.275 & -0.5 & 0.5 \\
\hline
\end{tabular}


Table 2 Variability of measured values for impedance sensor in corrosive atmosphere

\begin{tabular}{|c|c|c|c|c|c|c|c|c|c|}
\hline & \multicolumn{3}{|c|}{$100 \mathrm{~Hz}$} & \multicolumn{3}{|c|}{$1 \mathrm{kHz}$} & \multicolumn{3}{|c|}{$10 \mathrm{kHz}$} \\
\hline & Onoise & MIN & MAX & Onoise & MIN & MAX & Onoise & MIN & MAX \\
\hline air & - & - & - & - & - & - & 0.030 & -4.3 & 4.8 \\
\hline $0.001 \mathrm{M}$ AA, $52 \%$ RH & - & - & - & - & - & - & 0.017 & -61.1 & 29.1 \\
\hline $0.001 \mathrm{M}$ AA, $100 \% \mathrm{RH}$ & -0.002 & -13.8 & 21.7 & 0.001 & -13.4 & 20.9 & -0.002 & -86.3 & 90.2 \\
\hline $0.01 M$ AA, $52 \%$ RH & -0.006 & -24.8 & 18.6 & 0.008 & -24.6 & 19.7 & -0.002 & -19.5 & 19.0 \\
\hline $0.01 \mathrm{M} \mathrm{AA}, 100 \% \mathrm{RH}$ & 0.009 & -2.7 & 2.3 & -0.008 & -1.9 & 1.6 & 0.000 & -1.8 & 1.7 \\
\hline $0.1 \mathrm{M} \mathrm{AA}, 52 \% \mathrm{RH}$ & -0.579 & -1360.5 & 586.3 & 0.141 & -1306.8 & 545.5 & -0.003 & -178.2 & 80.6 \\
\hline
\end{tabular}

\section{CONCLUSION}

The advantage of the newly developed impedance sensor is its low production costs and higher sensitivity to changes in conditions in the monitored environment (changes in acetic acid concentration even at low relative humidity), unfortunately the measured data are more loaded with noise than data obtained by means of ER sensors. A new modification of the ER sensor design (design X) did not provide a higher sensitivity when measuring corrosion attack than commercially available variants (design $\mathrm{A}$ and $\mathrm{E}$ ), but it measures more accurately. The impedance sensor $(10 \mathrm{kHz})$ has shown higher ability to indoor atmospheres corrosion monitoring than ER sensors due to higher humidity and pollutants concentration sensitivity.

\section{ACKNOWLEDGEMENTS}

\section{The authors gratefully acknowledge the financial support by the Czech Ministry of Culture under} NAKI II programme (No. DG18P020W050).

\section{REFERENCES}

[1] RYHL-SVENDSEN, M. Corrosivity measurements of indoor museum environments using lead coupons as dosimeters. Journal of Cultural Heritage. 2008, vol. 9, pp. 285-293.

[2] NIKLASSON, A., JOHANSSON, L., SVENSSON, J. The influence of relative humidity and temperature on the acetic acid vapour-induced atmospheric corrosion of lead. Corrosion Science. 2008, vol. 50, pp. 3031-3037.

[3] NOVÁK, P. Preservation and restoration of lead and its alloys. In: Collective of Authors. Conservation and restoration of metals, 1 st ed. Brno: Technical Museum in Brno, 2011, pp. 526-544. (In Czech).

[4] PROSEK, T., Le BOZEC, N., THIERRY, D. Application of automated corrosion sensors for monitoring the rate of corrosion during accelerated corrosion tests. Materials and Corrosion. 2014, vol. 65, no. 5, pp. 448-456.

[5] PROSEK, T., KOURIL, M., DUBUS, M., TAUBE, M., HUBERT, V., SCHEFFEL, B., DEGRES, Y., JOUANNIC, M., THIERRY, D. Real-time monitoring of indoor air corrosivity in cultural heritage institutions with metallic electrical resistance sensors. Studies in Conservation. 2013, vol. 58, no. 2, pp. 117-128.

[6] BURIA, P. Measurement of corrosion processes. 2006. [viewed: 2021-04-04]. Available from: http://automa.cz/cz/casopis-clanky/mereni-koroznich-deju-2006 0831311 923/. (In Czech).

[7] JFE Techno-Research Corporation. Atmospheric Corrosion Monitoring by ACM Type Corrosion Sensor. [viewed: 2021-04-04]. Available from: https://www.jfe-tec.co.jp/en/tech-consul/fushoku16.html.

[8] ASTM G96-90.Standard Guide for Online Monitoring of Corrosion in Plant Equipment (Electrical and Electrochemical Methods). West Conshohocken, PA, USA: ASTM International, 2018.

[9] ISO 11844-2. Corrosion of metals and alloys - Classification of low corrosivity of indoor atmospheres - Part 2: Determination of corrosion attack in indoor atmospheres. Geneva, Switzerland: Technical Committee ISO/TC 156, 2020.

[10] ISO 11844-2. Corrosion of metals and alloys - Classification of low corrosivity of indoor atmospheres - Part 1: Determination and estimation of indoor corrosivity. Geneva, Switzerland: Technical Committee ISO/TC 156, 2020. 